\title{
Cuidado Parental e Apoio Social em Mães Residentes na Capital e Interior de Santa Catarina
}

\author{
Gabriela Dal Forno Martins \\ Samira Mafioletti Macarini \\ Mauro Luís Vieira \\ Universidade Federal de Santa Catarina \\ Virginia Azevedo Reis Sachetti \\ Faculdade Metropolitana de Guaramirim \\ Maria Lúcia Seidl-de-Moura \\ Universidade do Estado do Rio de Janeiro \\ Vera Silvia Raad Bussab \\ Universidade de São Paulo
}

\begin{abstract}
RESUMO
O objetivo do estudo foi investigar a relação entre cuidado parental e apoio social recebido por mães da capital e do interior de Santa Catarina. Participaram 50 mães de cada contexto, que responderam a duas escalas sobre cuidado parental. Uma que avalia a frequência de realização das práticas e outra que avalia o grau de importância atribuída às mesmas. Ambas as escalas eram compostas por itens relacionados às dimensões de cuidados primários e de estimulação. $\mathrm{O}$ apoio social foi avaliado através de uma terceira escala. Os resultados indicaram que: (a) existem diferenças significativas entre crenças sobre cuidado e apoio social das mães nos dois contextos, apontando para uma maior valorização dos cuidados primários e um maior apoio social entre as mães do interior; e (b) embora exista relação positiva entre apoio social e valorização dos cuidados de estimulação entre as mães da capital, o mesmo não se aplica às mães do interior; portanto, tal relação não pode ser aplicada indiscriminadamente, já que outras variáveis, possivelmente relacionadas ao contexto em que a mãe vive, influenciam suas crenças e práticas de cuidado.
\end{abstract}

Palavras-chave: cuidado parental; apoio social; contexto cultural.

\section{ABSTRACT \\ Childrearing Practices and Social Support Among Mothers Living in the Capital and Countryside of Santa Catarina}

The aim of this study was to investigate the relationships between mother's childrearing practices and their social support in the capital versus the countryside of Santa Catarina. In each cultural environment, fifty mothers participated in the study and were interviewed with two scales that evaluated their childrearing practices using two factors: primary care and stimulation. One scale evaluated the frequency of mother's childrearing practices and the other evaluated the degree of importance the mothers attributed to those practices. The mother's social support network was analyzed using a third scale. The results demonstrated that: (a) there was are significant differences in mother's reported childrearing practices and their social network between the cultural environments studied, suggesting that mothers from the countryside give more importance to primary care and have more extensive social support systems; and (b) even though there are some relationships between mother's childrearing practices and their social support, these relationships cannot be explained indiscriminately, once other variables, possibly related to mother's environment, interfere with their beliefs and practices regarding childcare..

Keywords: childrearing practices; social network; cultural environment.

A literatura contemporânea sobre desenvolvimento humano tem enfatizado a noção de que cada indivíduo se desenvolve em um ambiente específico, o qual possui uma organização particular de suas condições culturais. Nesse sentido, Harkness e Super (1992) criaram o conceito de nicho de desenvolvimento, des- 
crito em termos de três componentes: o ambiente físico e social no qual a criança vive, os costumes de cuidado e criação de crianças que são regulados culturalmente e a psicologia dos responsáveis ou conjunto de crenças parentais, nomeado etnoteorias parentais.

Os sistemas de crenças parentais ou etnoteorias podem ser definidos como conjuntos organizados de ideias que estão implícitos nas atividades da vida diária e nos julgamentos, escolhas e decisões que os pais tomam, funcionando como modelos ou roteiros para ações. Keller, Lohaus, Voelker, Elben e Ball (2003) relatam resultados de pesquisas demonstrando que as etnoteorias parentais são recursos que pais utilizam para formar conceitos sobre desenvolvimento, para autoavaliação da competência parental, para atribuição de sucesso ou fracasso ou para o estabelecimento de metas para si, para a criança e para a família. Desta forma, dentre outros aspectos relacionados à "parentalidade", as etnoteorias também abrangem: crenças de pais acerca do desenvolvimento infantil, metas de socialização e crenças sobre práticas de cuidado. As crenças que os responsáveis possuem a respeito do desenvolvimento infantil estão relacionadas às suas práticas de cuidado, conforme já evidenciado por outros autores (Meléndez, 2005; Seidl-de-Moura \& cols., 2004). As crenças sobre práticas de cuidado serão um dos focos do presente estudo, embora se tenha claro que os três tipos de cognições citadas estão estritamente vinculados (Keller, Lamm \& cols., 2006).

O estudo das crenças e práticas parentais tem sido realizado por alguns autores com base em três modelos culturais, em função de seus impactos nos processos de socialização e de desenvolvimento da criança: a) orientação cultural independente ou individualista; b) interdependente ou sociocêntrica (Keller, Borke, Yovsi, Lohaus \& Jensen, 2005; Keller, Hentschel \& cols., 2004) e c) autônomo-relacional (Kagitçibasi, 1996; 2005). A respeito desses três modelos, inicialmente, é preciso enfatizar que tais orientações culturais representam prioridades idealizadas culturalmente, o que não restringe a existência de outros tipos de orientações, combinações entre eles, além de variações intraculturais e interindividuais.

Os modelos culturais de independência e interdependência foram os primeiros descritos na literatura. O tipo de orientação individualista refere-se à construção do self como único e distinto, sendo priorizadas metas pessoais e com foco nas necessidades e direitos do indivíduo. Nesse modelo predominam as dimensões de autonomia e separação, característicos de so- ciedades urbanas pós-industriais com alto nível de escolaridade (Keller, Borke, Yovsi \& cols., 2005; Keller, Hentschel \& cols., 2004). O modelo de interdependência, por sua vez, concebe o self como fundamentalmente conectado aos demais membros do grupo em que está inserido, sendo priorizadas metas grupais e a focalização de papéis sociais, deveres e obrigações. Nesse tipo de orientação predominam as dimensões de heteronomia e relação (relatedness), características de ambientes rurais baseados em economia de subsistência (Keller, Borke \& cols., 2005; Keller, Hentschel \& cols., 2004). O terceiro modelo, proposto por Kagitçibasi (1996; 2005), denominado autônomo-relacional, compreende características combinadas de ambos os modelos anteriores. Esse tipo de orientação cultural engloba autonomia e relação (relatedness), em que o self é definido como autônomo quanto a sua ação e relacional quanto à proximidade interpessoal. Ele é característico de famílias de classe média, urbana e escolarizada em sociedades tradicionalmente interdependentes.

Keller $(2000 ; 2002 a ; 2002 b)$ afirma que os pais tendem a criar contextos de socialização para seus filhos coerentes com sua história pessoal e o contexto eco-cultural do qual são parte, fornecendo tanto cuidados primários como estimulação para facilitar o desenvolvimento psicológico em diferentes domínios, permitindo que as crianças adquiram sua matriz social e aprendam modos específicos de relacionamento social. A autora organizou os cuidados dispensados pelos pais à criança em categorias denominadas de sistemas parentais, os quais podem ser definidos como um conjunto de comportamentos geneticamente preparados e ativados pelas demandas ambientais com o objetivo de promover proximidade e conforto quando a criança está em risco real ou potencial.

Os cuidados primários, segundo Keller (2007), representam a parte filogenética mais antiga do cuidado parental, envolvendo abrigo, alimentação, higiene. A função psicológica básica deste sistema consiste em reduzir a estimulação estressante. Já os contatos corporais são definidos pelo contato físico corporal e por carregar a criança no colo com a função básica de promover calor emocional. A estimulação corporal é caracterizada por toques e movimentos que estimulam o desenvolvimento da coordenação motora e da percepção do corpo da criança em relação ao ambiente, contribuindo para a emergência de uma identidade corporal. Por sua vez, a estimulação por objetos propicia que a criança seja inserida no mundo dos objetos 
não pessoais e no ambiente físico em geral. Por fim, o sistema de trocas face a face envolve o contato do olhar e uso da linguagem.

Keller, Borke e Yovsi (2005) realizaram um estudo relacionando os sistemas parentais de cuidado citados acima a três diferentes grupos culturais: a) famílias rurais Camaronesas, b) famílias urbanas da Costa Rica e c) famílias urbanas da Alemanha. As famílias foram filmadas em situações de brincadeira livre, visando analisar as interações mãe-bebê segundo os sistemas parentais. Os resultados confirmaram que as mães alemãs possuem um estilo distal de parentalidade, com mais interações face a face e estimulação por objetos, e menos contato e estimulação corporal; o que tem sido relacionado, segundo os autores, com metas de socialização voltadas para a aquisição de autonomia e independência. As mães rurais Camaronesas, por outro lado, apresentaram um estilo mais proximal, com maior contato e estimulação corporal; interações que estão mais relacionadas com uma orientação cultural para interdependência. Por último, as mães da Costa Rica, também apresentaram um estilo proximal, porém com menos contato e estimulação corporal que as mães rurais Camaronesas, o que foi interpretado pelos autores como um estilo modificado, caracterizado como autônomo-relacional.

Enquanto esse último estudo procurou analisar as práticas dos cuidadores relacionando-as aos três modelos culturais (independente, interdependente e autônomo-relacional), outros autores tiveram como objetivo investigar mais especificamente as crenças sobre práticas de cuidado em pais de diferentes culturas. Por exemplo, Harkness e cols. (2001) verificaram que pais holandeses dão muita importância à prática de manter o bebê calmo e tranquilo. Eles valorizam o descanso, uma rotina regular e a higiene no cuidado com suas crianças. Ao contrário dos holandeses, pais americanos valorizam a estimulação. Segundo os autores, eles possuem ideias conflitantes acerca do descanso e da estimulação e, consequentemente relatam mais problemas em fazer as crianças dormir durante toda a noite.

Suizzo (2002) estudou crenças parentais sobre práticas de cuidado entre pais parisienses, através de uma escala construída pela própria autora, a qual possui três dimensões: "estimulação", "apresentação apropriada" e "responsividade e vínculo". A primeira dimensão foi a mais valorizada pelos pais, indicando que os mesmos acreditam na importância de proporcionar à criança diferentes estimulações, tais como interações diádicas e grupais, estimulação com brinquedos, através da linguagem, entre outros. Já a dimensão "apre- sentação apropriada" foi a segunda mais valorizada, porém obteve uma média também elevada, o que demonstra que os pais valorizam práticas que priorizam o bom comportamento e a higiene da criança em público. Por último, a dimensão "responsividade e vínculo", a qual contém itens relacionados à resposta dos pais às necessidades das crianças sem impor suas vontades, obteve uma média moderada.

No Brasil, Biasoli-Alves (1997; 2002) discute as mudanças nas crenças sobre criação de filhos, ocorridas ao longo dos séculos e afirma que a sociedade brasileira terminou o século XX com mudanças que incluem a ideia de "criança ideal", sendo tal crença influenciada pela disseminação dos conhecimentos científicos, vindos principalmente de médicos, psicólogos, pedagogos, entre outros. O acesso a esses conhecimentos, principalmente entre famílias residentes de cidades onde há maior influência da modernidade, segundo a autora, traz consigo o questionamento de antigos valores e a intensa procura por novos padrões de comportamento.

Alguns estudos brasileiros foram desenvolvidos com o objetivo de identificar a importância atribuída às dimensões de crenças verificadas por Suizzo (2002). Por exemplo, Ruela (2006) estudou crenças sobre práticas de cuidado e metas de socialização entre mães e avós de uma comunidade rural no Rio de Janeiro, enquanto Kobarg (2006) estudou semelhanças e diferenças nas crenças e práticas de cuidados de mães dos contextos rural e urbano em Santa Catarina. Piovanotti (2007), por sua vez, investigou práticas de cuidado parental valorizadas por mães primíparas residentes em um contexto urbano na capital do estado de Santa Catarina. Os resultados desses estudos em conjunto indicaram que mães do contexto rural e as mães urbanas com baixo nível de escolaridade valorizaram a apresentação apropriada do bebê em público, seguida pela estimulação; enquanto as mães urbanas com alto nível de escolaridade valorizaram mais a estimulação do que a apresentação.

Além das variáveis sociodemográficas e de aspectos culturais específicos de cada contexto, o apoio social recebido pelas mães tem sido apontado como um fenômeno que pode afetar a relação mãe e filho de muitas formas (Jennings, Stagg \& Connors, 1991). Assim como todas as interações humanas, a relação mãe-criança ocorre em um contexto social concreto, sendo especialmente importantes as relações com a família, amigos, parentes e vizinhos, uma vez que estes formam o suporte social imediato que a mãe dispõe para cuidar da criança. Por apoio social compreende-se ter outras pessoas que possam oferecer 
ajuda, encorajamento e conselhos. O apoio social fornece informações que levam o indivíduo a acreditar que é cuidado, amado e que pertence a um grupo com obrigações mútuas (Cohen \& Wills, 1985; Griep, Chor, Faerstein, Werneck \& Lopes, 2005).

Crittenden (1985) aponta que a rede de apoio social que a mãe dispõe tem efeito direto sobre o cuidado destinado à criança. Quanto maior o suporte social recebido, maior tende a ser a capacidade de enfrentar as mudanças na vida e menor a sensação de ameaça ou perda, funcionando como um recurso ambiental no processo de enfrentamento de situações difíceis (Cohen \& Wills, 1985; Griep \& cols. 2005). Redes de apoio social, que possuem papel de suporte em situações difíceis, refletem na relação com o filho e são associadas a indicadores de cuidados adequados, como calor, respostas às necessidades da criança e satisfação do papel parental. Na criança, são associadas ao desenvolvimento de vínculos seguros, interação cooperativa e competências cognitivas.

De maneira geral, os resultados de pesquisas e ideias expostas até então indicam que as crenças e práticas parentais sobre cuidado apresentam-se de maneira diferenciada em contextos culturais específicos, os quais variam entre dois pólos protípicos: a independência e a interdependência. Além disso, as crenças e práticas sobre cuidado são também moduladas por variáveis específicas da amostra, como a escolaridade e o apoio social recebido pelos cuidadores. Diante disso, o principal objetivo deste estudo foi investigar se existe relação entre as crenças e práticas de cuidado e o apoio social recebido pelas mães residentes no interior e na capital do estado de Santa Catarina. Esperava-se encontrar uma relação entre o apoio social e as crenças e práticas características de modelos mais interdependentes, já que, como demonstra a literatura (Keller, Borke, Yovsi \& cols., 2005; Keller, Hentschel \& cols., 2004), nesses contextos há um predomínio de metas grupais e a focalização de papéis sociais, deveres e obrigações.

Como objetivos específicos, o estudo pretendeu identificar se existem diferenças entre as crenças e práticas de cuidado das mães residentes nos dois contextos, assim como identificar se existem diferenças no apoio social recebido por elas. Tinha-se como hipótese que no interior seriam identificadas crenças e práticas mais próximas de um modelo interdependente (como o contato corporal), uma vez que as cidades pesquisadas neste estudo possuem características comuns a contextos interdependentes estudados por outros autores, como a economia baseada na agricultura e baixo nível socioeconômico e de escolaridade.
Por outro lado, na capital, esperava-se que as crenças e práticas dos pais seriam mais próximas de um modelo independente (estimulação face a face e por objetos), já que a cidade estudada possui um maior nível de urbanização, as mães pertencem em geral à classe média e são escolarizadas. Com relação ao apoio social, esperava-se que no interior ele seria mais elevado, uma vez que em cidades de baixa população, é comum que a maioria dos moradores se conheça e que possam contar uns com os outros.

\section{MÉTODO}

Para a realização do presente estudo foram utilizados instrumentos construídos pelos próprios pesquisadores, bem como medidas já validadas em amostras brasileiras. Dessa forma, realizou-se uma etapa preliminar de submissão das escalas ainda não validadas a um procedimento de análise inicial, visando medir a validade e a confiabilidade das mesmas. No entanto, embora ao longo do artigo esse procedimento seja mencionado, será dada ênfase aos resultados referentes à avaliação das crenças maternas sobre cuidados com crianças e sua relação com o apoio social.

\section{Participantes}

Participaram da etapa de adaptação das escalas ao contexto brasileiro 250 mães residentes em cinco contextos diferentes (50 em Florianópolis, 75 no norte catarinense, 50 no oeste catarinense, 50 na cidade do Rio de Janeiro e 25 em Belém), acima de 18 anos com, pelo menos, um filho entre zero e 6 anos na época da coleta de dados.

No estudo principal participaram 100 mães de dois contextos distintos (50 mães residentes na capital e 50 mães residentes no interior do Estado de Santa Catarina), com idade acima de 21 anos, com pelo menos um filho entre zero e seis anos de idade na época da coleta de dados (criança-foco). O Estado de Santa Catarina está localizado na Região Sul, sendo a capital Florianópolis, município de colonização açoriana, o qual possuía população em 2001 de 342.315 e estimada em 2006 de 406.564 habitantes (Instituto Brasileiro de Geografia e Estatística, 2006). A principal atividade econômica da cidade está ligada ao turismo e comércio (Governo do Estado de Santa Catarina, 2006).

As duas cidades pesquisadas no interior do estado, Corupá e Schroeder, foram agrupadas para fins de coleta de dados em um único contexto, por serem semelhantes em vários aspectos. Ambas são predominantemente de colonização alemã e possuem até 13.000 habitantes (IBGE, 2006). Além disso, a economia dos dois municípios gira em torno do cultivo e 
industrialização da banana, sendo Corupá o principal produtor do Estado (Governo do Estado de Santa Catarina, 2006).

\section{Instrumentos}

Foram utilizadas duas escalas elaboradas para a presente pesquisa, visando avaliar os cuidados parentais, as quais passaram por uma adaptação na etapa preliminar. Além disso, utilizou-se uma terceira escala para avaliar o apoio social recebido pelas mães, a qual já havia sido submetida a um processo de adaptação ao contexto brasileiro. Também foi aplicado um questionário para investigar dados sociodemográficos. Abaixo seguem mais informações sobre cada um dos instrumentos:

1) Questionário sociodemográfico: roteiro estruturado referente a variáveis do tipo: idade, número de filhos, local de residência, tipo de família, escolaridade e renda familiar.

2) “Atividades Realizadas pela Mãe" e "Importância Atribuída às Atividades": os instrumentos originais foram elaborados pelo núcleo de pesquisa "Interação social e desenvolvimento" coordenado pela Prof ${ }^{\mathrm{a}}$. Maria Lúcia Seidl-de-Moura, do Instituto de Psicologia da Universidade do Estado do Rio de Janeiro UERJ. Foram inicialmente elaborados para abranger os cinco sistemas parentais descritos por Keller (2000, 2002b, 2007): cuidados primários, contato corporal, estimulação corporal, estimulação com objetos e trocas face a face.

As duas escalas são compostas por 25 afirmativas idênticas e distribuídas nas cinco dimensões descritas acima. O instrumento denominado "Atividades Realizadas pela Mãe" avalia a frequência de realização efetiva das práticas por parte da mãe (por exemplo, se a mãe carrega a criança no colo), segundo uma escala de cinco pontos que vai desde 1 (nunca) até 5 (sempre). Já o instrumento denominado "Importância Atribuída às Atividades Realizadas" investiga valores e crenças, indicando uma avaliação da mãe sobre a importância de cada prática (por exemplo, se é importante carregar a criança no colo), segundo uma escala de cinco pontos que vai desde 1 (pouco importante) até 5 (muito importante).

3) Escala de aferição de apoio social: originalmente elaborada por Sherbourne e Stewart (1991) e traduzida, adaptada e validada para o Brasil pelo Es- tudo Pró-Saúde (Griep \& cols., 2005). É uma escala tipo Likert, de 5 pontos $(1=$ nunca até $5=$ sempre $)$, que avalia a frequência com que a mãe possui a sua disposição laços sociais e recursos de outras pessoas de sua rede em caso de necessidade. Esta versão da escala mantém o conjunto original de perguntas (19 itens) e inclui três fatores: apoio material (por exemplo, se você conta com alguém que a ajude, se ficar de cama), emocional/informação (por exemplo, se há alguém para dar bons conselhos em situação de crise) e interação social positiva/apoio afetivo (por exemplo, se você tem alguém com quem fazer coisas agradáveis).

\section{Procedimentos de coleta dos dados}

Após o projeto ter sido aprovado pelo Comitê de Ética em Pesquisas com Seres Humanos (protocolos $n^{\circ} 166 / 06$ e 239/06), iniciou-se a coleta dos dados. Na capital, as mães foram contatadas através de creches e de indicações das próprias mães participantes. No interior, além das creches, muitas mães foram contatadas em unidades de saúde. Os instrumentos foram aplicados individualmente, na forma de entrevistas estruturadas, no local de preferência da mãe, predominando seu domicílio, universidade federal e as unidades de saúde.

\section{Análise dos dados}

As duas escalas adaptadas na etapa preliminar foram avaliadas através de uma análise fatorial e do cálculo de consistência interna dos fatores, sendo utilizado para tanto o programa estatístico SPSS (versão 11.5). Foram realizados os seguintes procedimentos nesta ordem:

1) extração dos componentes principais, através do qual se verificou a adequação da matriz quanto aos pressupostos necessários à análise multivariada, utilizando-se o indicador Kaiser-Meyer-Olkin (KMO) para averiguar a adequação da fatorabilidade. A extração dos componentes principais permitiu também que se definissem o número de fatores, segundo três critérios (Dancey \& Reidy, 2006; Suizzo, 2002): a) eigenvalue maior que 1 ; b) teste scree plot de Catell; e c) interpretação teórica; 2) rotação varimax para extrair os fatores, sendo excluídos itens que pesaram em mais de um fator ou itens com carga fatorial inferior ou igual a 0,30 ; e 3 ) avaliação da consistência interna de cada fator através do cálculo do índice alpha de Cronbach. 
No estudo principal, foram realizadas análises descritivas como cálculos de frequência, média e porcentagens. Além disso, foram realizados cálculos de comparação de médias (Teste-t e ANOVA) e correlações (Pearson).

\section{RESULTADOS}

\section{1) Etapa preliminar de adaptação das escalas de cuidado parental}

O processo de análise fatorial utilizado na adaptação das duas escalas apontou a existência de dois fatores (e não mais as cinco dimensões sugeridas na versão da escala original), com nova distribuição dos itens, que passaram a ser denominados de cuidados primários e estimulação. Como as duas escalas possuem as mesmas afirmações e investigações do ponto de vista da prática (escala 1) e da importância atribuída (escala 2), foram excluídos da versão final de ambas os itens que pesaram em mais de um fator em pelo menos uma escala, os itens que pesaram em um fator em uma escala e em outro fator na outra escala e os itens com carga fatorial inferior ou igual a $0,30 \mathrm{em}$ pelo menos uma das escalas.

Os cinco primeiros itens da dimensão de cuidados primários permaneceram os mesmos da versão original da escala, acrescidos de três itens anteriormente classificados como contato corporal (carregar no colo; ter sempre por perto; evitar que se acidente). Os demais itens, que eram relacionados às dimensões de estimulação corporal, estimulação com objetos e trocas face a face, foram agrupados em um único fator (estimulação), exceto os itens excluídos (abraçar e beijar; dormir junto na rede ou cama; fazer cócegas; fazer massagem; deixar livre para correr, nadar, trepar; dar brinquedos; conversar).

As dimensões da escala de "Atividades realizadas pela mãe" obtiveram índices alpha de Cronbach satisfatórios, sendo 0,70 para a dimensão cuidados primários e 0,82 para a dimensão cuidados de estimulação. Por sua vez, as dimensões da escala de "Importância atribuída às atividades" obtiveram índices alphas bastante semelhantes aos anteriores e, portanto, também satisfatórios, sendo 0,68 para a dimensão cuidados primários e 0,81 para os cuidados de estimulação.

\section{2) Crenças de mães sobre cuidados com crianças e sua relação com o apoio social}

\section{Caracterização sociodemográfica da amostra}

As 50 mães residentes em Florianópolis apresentaram idade variando entre 21 e 49 anos, com média de $32,14(D P=6,37)$. A maioria delas possuía apenas um filho $(n=30)$, na época da coleta de dados era casada ou vivia em união estável $(n=42)$ e foi criada na zona urbana $(n=42)$. Já as 50 mães residentes no interior apresentaram idade variando entre 21 e 38 anos, com média de 29,16 $(D P=3,82)$. A maioria delas também tinha apenas um filho $(n=28)$, era casada ou vivia em união estável $(n=46)$ e foi criada na zona rural $(n=$ 28). As crianças-foco da capital apresentaram uma média de idade, em meses, de 37,90 $(D P=15,22)$, enquanto que as crianças do interior apresentaram uma média de 39,56 meses $(D P=22,44)$.

O nível de escolaridade foi avaliado através de uma escala de 9 pontos, em que 1 correspondia a mães não alfabetizadas e 9 a mães pós-graduadas. As mães da capital apresentaram uma média de 7,2 $(D P=$ $2,09)$, enquanto as mães do interior apresentaram uma média de 5,8 $(D P=1,78)$. Por sua vez, a renda familiar mensal foi medida através de uma escala de 14 pontos, em que 1 representava uma renda de até cem reais e 14 uma renda acima de quatro mil reais. A média entre as mães da capital foi de $11,88(D P=2,46)$ e entre as mães do interior de $11,46(D P=12,81)$. Por fim, verificou-se que a ampla maioria das mães, tanto na capital como no interior, possuíam trabalho remunerado ( $n=39$ e 43, respectivamente).

Além das estatísticas descritivas, foram realizadas análises de comparação de média (teste $t$ ) e de associação de variáveis (qui-quadrado) visando comparar as variáveis sociodemográficas nos dois contextos. Os resultados indicaram que as mães do interior são significativamente mais jovens $(t(98)=0,298 ; p<0,01)$ e as mães residentes na capital têm, significativamente, maior escolaridade $(t(98)=3,60 ; p<0,001)$. Foi verificado também que as mães da capital foram criadas predominantemente na zona urbana e as mães do interior foram criadas predominantemente na zona rural $\left(\chi^{2}=17,36 ; g l=1 ; p<0,01\right)$.

\section{Atividades de cuidado realizadas e valorizadas}

Os resultados referentes às duas escalas sobre cuidados estão expostos na Tabela 1 . O teste $t$ evidenciou diferenças significativas tanto dentro de cada contexto, quanto entre os contextos (coluna à direita da Tabela 1). 
TABELA 1

Resultados da Escala de Atividades Realizadas e da Escala de Importância Atribuída às Atividades Realizadas: Média, Desvio-padrão e Diferenças Significativas no Contexto e entre Contextos

\begin{tabular}{|c|c|c|c|}
\hline Escalas & $\begin{array}{l}\text { Capital } \\
M(D P)\end{array}$ & $\begin{array}{l}\text { Interior } \\
M(D P)\end{array}$ & $\mathrm{t}(98)$ \\
\hline \multicolumn{4}{|l|}{ Atividades Realizadas } \\
\hline Cuidados primários & $4,60(0,33)$ & $4,67(0,30)$ & 1,17 \\
\hline Estimulação & $4,05(0,67)$ & $4,06(0,55)$ & 0,10 \\
\hline$t(49)$ & $-5,63^{* *}$ & $-6,57^{* *}$ & \\
\hline \multicolumn{4}{|l|}{ Importância Atribuída às Atividades } \\
\hline Cuidados primários & $4,52(0,29)$ & $4,71(0,30)$ & $3,13^{\star *}$ \\
\hline Estimulação & $4,36(0,35)$ & $4,43(0,53)$ & 0,81 \\
\hline$t(49)$ & $-2,57^{\star *}$ & $-3,65^{\star *}$ & \\
\hline
\end{tabular}

Tanto na capital como no interior, em termos de práticas, as mães relataram realizar significativamente mais cuidados primários do que cuidados voltados para a estimulação do desenvolvimento. Também, em geral, as mães dão maior importância às práticas de cuidados primários do que às práticas de estimulação. Estes dados sugerem que há relação entre a prática, enquanto comportamento, e a valorização da mesma em termos de importância. Além disso, verifica-se que apesar de os cuidados primários serem mais valorizados entre as mães da capital e do interior, a comparação entre estes contextos mostrou que as mães do interior atribuem significativamente maior importância aos cuidados primários do que as mães da capital.

Foram também realizados cruzamentos entre os dados sócio-demográficos e as dimensões das escalas por contexto. $\mathrm{Na}$ capital, foi verificado que quanto maior a escolaridade das mães, menos elas realizam cuidados primários $(r=-0,36 ; p=0,01)$ e mais valo- rizam os cuidados de estimulação $(r=0,32 ; p<0,05)$. Além disso, quanto maior a idade e a renda das mães, mais elas são favoráveis às práticas de estimulação $(r=0,41 ; p<0,01$ e $r=0,29 ; p<0,05$, respectivamente). Já no interior, os resultados indicaram que quanto maior a idade da criança-foco, mais as mães realizam atividades de estimulação $(r=0,44 ; p<0,01)$. Por outro lado, houve correlação negativa entre número de filhos e a realização de atividades de estimulação $(r=-0,31 ; p<0,05)$, sugerindo que um número maior de filhos implica em menos tempo disponível para realizar atividades de estimulação, uma vez que provavelmente há maior necessidade de investimento em cuidados primários.

\section{Apoio Social}

Os resultados referentes ao apoio social, por contexto e entre os contextos são apresentados na Tabela 2.

TABELA 2

Resultados da Escala de Apoio Social: Média, Desvio-padrão e Comparação do Escore Total e das Dimensões no Contexto e entre os Contextos

\begin{tabular}{cccc}
\hline Apoio Social & Capital & Interior & $t(98)$ \\
& $M(D P)$ & $M(D P)$ & \\
\hline Afetivo/interação social positiva & $4,21(0,63)$ & $4,51(0,65)$ & $2,29^{*}$ \\
Emocional/informação & $3,98(0,71)$ & $4,24(0,88)$ & 1,58 \\
Material & $3,92(1,01)$ & $4,54(0,73)$ & $3,50^{* *}$ \\
\hline $\mathrm{F}$ & $\mathrm{F}(1,69)=4,84^{*}$ & $\mathrm{~F}(1,63)=6,64^{* *}$ & \\
\hline${ }^{*} p<0,05 ;{ }^{* *} p<0,01$ & &
\end{tabular}

Visando avaliar as diferenças entre as médias das três dimensões de apoio em cada contexto, foi realizada uma ANOVA de um fator para medidas repetidas. $\mathrm{O}$ teste de efeito dentre os sujeitos Greenhouse-
Geisser revelou diferenças significativas entre as três dimensões na capital e no interior. Por sua vez, o teste de comparação dos pares de médias (Bonferroni) revelou que as mães da capital recebem significativa- 
mente mais apoio social na dimensão afetivo/interação social positiva do que nas dimensões emocional/informação $(p<0,05)$, e material $(p<0,05)$. No interior, as mães recebem significativamente mais apoio social na dimensão material do que na dimensão emocional/informação $(p<0,05)$, e significativamente mais apoio na dimensão afetivo/interação social positiva do que na dimensão emocional /informação $(p<0,05)$.

Ao comparar os dois contextos, os resultados indicaram que as mães do interior apresentam médias maiores em todas as dimensões da escala de apoio social do que mães residentes na capital. No entanto, essas diferenças foram estatisticamente significativas somente na dimensão apoio material.

\section{Relações entre cuidado dispensado à criança $\mathrm{e}$ apoio social recebido}

Visando investigar a relação existente entre o cuidado parental e o apoio social recebido pelas mães, as dimensões dos três instrumentos foram correlacionadas em cada contexto separadamente. Os resultados somente foram significativos na capital e indicaram que mães que recebem maior apoio na dimensão emocional/informação, realizam menos atividades de cuidados primários $(r=-0,295, p<0,05)$, talvez porque tenham ajuda para compartilhar os cuidados com a criança. Também se verificou que quanto mais apoio social recebido nas dimensões afetivo/interação social positiva $(r=0,408, p<0,01)$, material $(r=0,391$, $p<0,01)$ e na dimensão geral de apoio $(r=0,395$, $p<0,01)$, maior importância é atribuída às atividades de estimulação. Estes resultados sugerem que quanto mais suporte social as mães possuem, mais valorizam práticas de estimulação e menos ficam restritas a cuidados básicos.

\section{DISCUSSÃO}

O objetivo principal desse estudo foi investigar se existe relação entre as crenças e práticas de cuidado e o apoio recebido por mães de contextos distintos: capital e interior. Os resultados indicaram que há relação entre cuidado parental e apoio social somente entre as mães da capital. Além disso, as hipóteses inicialmente formuladas a respeito das diferenças entre os contextos foram em parte confirmadas.

Quanto às crenças e práticas de cuidado, os resultados indicaram que mães do interior dão maior importância aos cuidados primários do que as mães da capital, não sendo encontradas outras diferenças significativas. Esse resultado era esperado, uma vez que a dimensão cuidados primários engloba itens referentes aos sistemas de cuidados primários e contato corporal (Keller, 2002b). Este último sistema tem sido associado em outros estudos a modelos culturais mais interdependentes (Keller, Borke \& Yovsi, 2005; Keller \& Lamm, 2005; Keller, Yovsi \& cols., 2004). Os mesmos estudos demonstram que em modelos culturais mais independentes há um predomínio de cuidados que envolvam interações face a face e estimulação por objetos, os quais estavam contemplados na dimensão estimulação dos instrumentos utilizados neste estudo. No entanto, conforme verificado, entre as mães da capital esse tipo de cuidado não foi mais realizado e valorizado do que entre as mães do interior.

Esse resultado pode ser explicado a partir das características sociodemográficas e culturais das mães residentes no interior. Embora seja um contexto menos urbanizado que a capital e tenha como principal atividade econômica a agroindústria, as mães do interior possuem renda mensal semelhante às mães da capital, escolaridade mais baixa, porém a maioria $(n=$ 27) possui pelo menos o segundo grau completo ou superior incompleto e possuem um trabalho remunerado, o qual se situa geralmente em cidades de maior população próximas. Além disso, um aspecto importante entre as mães do interior refere-se a sua origem étnica alemã, como já mencionado antes, cultura essa descrita na literatura como sendo predominantemente independente (Keller, Borke \& Yovsi, 2005; Keller \& Lamm, 2005; Keller, Yovsi \& cols., 2004). Essas características, juntamente com os resultados encontrados a respeito das crenças e práticas das mães e do apoio social recebido, indicam que provavelmente o modelo predominante no interior seja o autônomorelacional, o qual, segundo Kagitçibasi (1996; 2005), engloba autonomia e relação, em que o self é definido como autônomo quanto a sua ação e relacional quanto à proximidade interpessoal.

Outro resultado verificado entre as mães do interior refere-se a uma correlação positiva entre a idade da criança e a realização de atividades de estimulação, a qual sugere que, com o crescimento da criança, há uma menor necessidade de cuidados primários e a possibilidade de um maior investimento em atividades de estimulação do desenvolvimento. A crença que poderia estar subjacente a essa correlação é a de que crianças mais velhas já possuem maturação para responder de maneira mais específica aos diferentes estímulos, e que crianças menores necessitariam mais dos cuidados primários. Nesse sentido, verifica-se que as crenças que os cuidadores possuem a respeito do 
desenvolvimento infantil estão relacionadas às suas práticas de cuidado, conforme já evidenciado por outros autores (Meléndez, 2005; Seidl-de-Moura \& cols., 2004). Esse resultado aponta ainda para a importância de se realizarem trabalhos com os pais que envolvam a transmissão de informações sobre o desenvolvimento infantil, como forma de promoção do desenvolvimento e da saúde das crianças.

Algumas correlações entre crenças e práticas de cuidado e variáveis sóciodemográficas foram também verificadas entre as mães da capital. Quanto maior a escolaridade, idade e renda das mães, mais elas valorizaram práticas de estimulação. A valorização da estimulação tem sido associada à escolaridade das mães em diversos estudos (Kobarg, 2006; Piovanotti, 2006; Suizzo, 2002). No caso deste estudo, os cuidados de estimulação correlacionaram-se à escolaridade somente na capital, embora, como já discutido, as mães do interior realizavam e valorizavam tais cuidados tanto quanto as mães da capital.

Ainda é preciso destacar que tais correlações ocorreram apenas com a dimensão valorativa dos cuidados de estimulação e não com as atividades realizadas propriamente. Isso indica que um maior acesso a informações proporcionadas pela escolaridade e renda das mães permite que elas reflitam sobre a interação com os filhos, incluindo no seu repertório de crenças a valorização da estimulação. Isso pode ser explicado pelo processo histórico a respeito das mudanças nas crenças sobre cuidados parentais no contexto brasileiro no final do século XX e início do século XXI, discutido por Biasoli-Alves (1997; 2002). Dessa forma, pode-se pensar que a correlação verificada na capital reflete de alguma forma esta maior preocupação dos pais com o desenvolvimento, sem que isso implique ainda em práticas consolidadas como mais e menos adequadas. Novas pesquisas podem concentrar seus esforços em investigar a relação entre as crenças dos pais e sua prática, no sentido de identificar fatores decisivos na escolha desta última.

Diferenças entre interior e capital foram verificadas também com relação ao apoio social recebido pelas mães. Mães do interior apresentaram, de maneira geral, maiores médias com relação ao apoio social recebido, o que já era esperado, uma vez que se hipotetizou que o contexto do interior possui características mais próximas de um modelo interdependente no que diz respeito à proximidade pessoal, predominando metas grupais e a focalização de papéis sociais, deveres e obrigações. Em cidades de baixa população, é comum que a maioria dos moradores se conheça e que possam contar uns com os outros, principalmente no que diz respeito a necessidades materiais. Dessa for- ma, houve no interior um predomínio das dimensões material e afetivo/interação social.

$\mathrm{Na}$ capital, embora o apoio afetivo/interação social também tenha sido elevado, o apoio material obteve a menor média. Durante as entrevistas com as mães da capital, estas relatavam que sua principal fonte de apoio era o esposo, que tinha como papel principal fornecer apoio afetivo e ser companheiro nas atividades de lazer, mas não ajudar nas tarefas diárias ligadas ao ambiente doméstico, as quais fazem parte da dimensão material. Em termos de proximidade social, portanto, a capital parece aproximar-se de modelos culturais mais independentes, em que são priorizadas metas pessoais, focando-se nas necessidades e direitos do indivíduo. Isso não significa, no entanto, afirmar que nesses modelos não há apoio social, mas há indícios de que o apoio nesse caso é fornecido por pessoas bastante próximas e por um número menor delas, focado principalmente no afeto e no lazer. Pode-se pensar também que na capital as mulheres acabam mais sobrecarregadas em termos de trabalho, pois além de a maioria possuir um emprego fora de casa, pode contar com menos ajuda nas tarefas domésticas quando não as podem realizar.

Apesar de as mães do interior receberem maior apoio social do que as mães da capital, esta dimensão foi correlacionada com as crenças e práticas parentais somente neste último contexto. É possível que exatamente pelo fato de o apoio social ser maior no interior, seu efeito parece se apresentar de maneira mais crítica na capital. Destaca-se também o fato de esta correlação ter ocorrido positivamente com a valorização dos cuidados de estimulação, uma vez que o apoio social é característico de contextos mais interdependentes e os cuidados de estimulação característicos de contextos mais independentes. No entanto, esse resultado faz sentido tendo em vista o predomínio de uma orientação autônomo-relacional nesse grupo estudado. Assim, o apoio social parece permitir que as mães reflitam sobre seu papel enquanto cuidadoras, dando ênfase a cuidados que tenham por objetivo estimular o desenvolvimento da criança. Talvez, na capital, os questionamentos das mães sobre como educar da maneira mais adequada os filhos façam com que elas necessitem de maior apoio emocional, afetivo, de interação, visando dividir sua ansiedade e preocupação. Biasoli-Alves (2002) também afirma que um fator importante, presente na modernidade, refere-se à escolarização cada vez mais precoce das crianças. Este aspecto tem como consequência a profissionalização do "cuidar de crianças", não implicando a perda do lugar da criança na família, mas uma intensa preo- 
cupação dos pais com o seu desenvolvimento. Com isso, pode-se concluir que o apoio social na capital é um fator de proteção tanto para a família quanto para a criança.

\section{CONSIDERAÇÕES FINAIS}

Com base nos resultados obtidos no presente estudo, é possível destacar três contribuições principais. A primeira refere-se às diferenças nas crenças e práticas parentais verificadas entre os contextos. O estudo confirmou que o cuidado parental, embora tenha algumas características universais, é influenciado diretamente pelo contexto cultural específico em que as mães estão inseridas. Nesse sentido, as práticas das mães têm a função de proporcionar à criança a aprendizagem das habilidades específicas que ela necessitará para inserir-se no contexto social em que vive. A segunda contribuição refere-se à relação encontrada entre o apoio social e o cuidado parental. Embora o apoio possa ser considerado um fator de proteção para o desenvolvimento da criança, tal relação deve sempre ser compreendida no contexto em que ocorre. No interior, onde os níveis de apoio social são em geral mais altos do que na capital, pode-se captar indícios de que outras variáveis influenciem de maneira mais forte as crenças e práticas de cuidado, como, por exemplo, a idade da criança e o número de filhos.

Essas duas primeiras contribuições têm implicações diretas nas práticas a serem exercidas pelos profissionais que trabalham com famílias e crianças. Dessa forma, uma terceira implicação do estudo relaciona-se à importância de as intervenções levarem em conta as especificidades do contexto em que se atua, incluindo a forma de organização social, as características socioeconômicas da população, entre outros. Programas de orientação para as famílias mostram-se necessários, tanto para fornecer informações sobre o desenvolvimento infantil, quanto para problematizar práticas que venham a permitir que as crianças desenvolvam habilidades funcionais no contexto em que vivem. É preciso devolver às famílias a especialidade no cuidado às crianças, uma vez que a história da espécie humana mostra que os pais são aqueles mais habilitados para cuidar de seus próprios filhos.

Por fim, algumas limitações do estudo podem ser evidenciadas, assim como a necessidade de outros estudos na área. Uma limitação refere-se ao acesso da dimensão das práticas parentais através de questionários e não de observação. Crenças são facilmente acessadas através dos relatos dos pais, no entanto, as práticas são mais adequadamente avaliadas por meio da observação da interação cuidador-criança (Harkness \& Super, 1992). Dessa forma, alguns resultados podem não ter sido verificados em função desta limitação. Novos estudos devem ser realizados através da observação e podem focalizar a relação entre crenças e práticas parentais, visando verificar que outros aspectos estão influenciando o comportamento dos pais, além de suas crenças. Como foi verificada relação entre apoio e cuidado parental, é necessário agora investigar como essa relação ocorre, quem são as pessoas que fornecem apoio e quais as consequências do apoio recebido pela mãe no desenvolvimento da criança. Novas investigações do grupo de pesquisa buscarão trazer uma contribuição nesse sentido.

\section{REFERÊNCIAS}

Biasoli-Alves, Z. M. M. (1997). Famílias brasileiras do século XX: Os valores e as práticas da educação da criança. Temas em Psicologia, 3, 33-40.

Biasoli-Alves, Z. M. M. (2002). A questão da disciplina na prática de educação da criança, no Brasil, ao longo do século XX. Veritati, 2(2), 243-259.

Cohen, S., \& Wills, T. A. (1985). Stress, social support, and the buffering hypothesis. Psychological Bulletin, 98(2), 310-357.

Crittenden, P. M. (1985). Social networks, quality of child rearing, and child development. Child Development, 56, 1298-1313.

Dancey, C. P., \& Reidy, J. (2006). Estatística sem matemática para psicologia. Porto Alegre: Artmed.

Governo do Estado de Santa Catarina (2006). Municípios. Retirado em 20 de julho de 2007, de http://www.sc.gov/ conteudo/municipios.

Griep, R. H., Chor, D., Faerstein, E., Werneck, G. L., \& Lopes, C. S. (2005). Validade do constructo de escala de apoio social do Medical Outcomes Study adaptada para o português no Estudo Pró-Saúde. Cadernos de Saúde Pública, 21(3), 703-714.

Harkness, S., \& Super, C. M. (1992). Parental ethnotheories in action. Em I. S. Siegel, A. V. McGillicudy-DeLisi \& J. Goodnow (Orgs.), Parental belief systems: The psychological consequences for children (pp. 373-392). Hillsdale, NJ/Hove, UK: Lawrence Erlbaum.

Harkness, S., Super, C. M., Axia, V., Eliaz, A., Palacios, J., \& Welles-Nyström, B. (2001). Cultural pathways to sucessful parenting. International Society for the Study of Behavioral Development Newsletter, 38(1), 9-13.

Instituto Brasileiro de Geografia e Estatística. (2006). Cidades. Retirado em 20 de julho de 2007, de http:/www.ibge.gov.br

Jennings, K. D., Stagg, V., \& Connors, R. E. (1991). Social networks and mothers' interactions with their preschool children. Child Development, 62, 966-978.

Kagitçibasi, Ç. (1996) The autonomous-relational self: A new synthesis. European Psychologist, 1(3), 180-186.

Kagitcibasi, C. (2005). Autonomy and relatedness in cultural context: Implications for self and family. Journal of CrossCultural Psychology, 36(4), 403-422. 
Keller, H. (2000). Human parent-child relationships from an evolutionary perspective. American Behavioral Scientist, 43(6), 957-969.

Keller, H. (2002a). Culture and development: Developmental pathways to individualism and interrelatedness. Em W. J. Lonne, D. L. Dinnel, S. A. Hayes \& D. N. Sattler (Orgs.). Online readings in psychology and culture (Unidade 11, Cap. 1). Center for Cross-Cultural Research. Western Washington University, Bellingham, Washington. Retirado em 07 de julho de 2007, de http://www.wwu.edu/ culture.

Keller, H. (2002b). Development as the interface between biology and culture: A conceptualization of early ontogenetic experiences. Em H. Keller, Y. H. Poortinga \& A. Schölmerich (Orgs.), Between culture and biology: Perspectives on ontogenetic development (pp. 215-235). Cambridge: Cambridge University Press.

Keller, H. (2007). Cultures of infancy. Mahwah, NJ: Lawrence Erlbaum.

Keller, H., Borke, J., \& Yovsi, R. (2005) Cultural orientations and historical changes as predictors of parenting behaviour. International Journal of Behavioral Development, 29(3), 229237.

Keller, H., Borke, J., Yovsi, R., Lohaus, A., \& Jensen, H. (2005). Cultural orientations and historical changes as predictions of parenting behavior. International Journal of Behavioral Development, 29(3), 229-237.

Keller, H., Hentschel, E., Yovsi, R. D., Lamm, B., Abels, M., \& Haas, V. (2004). The psycho-linguistic embodiment of parental ethnotheories: A new avenue to understanding cultural processes in parental reasoning. Culture \& Psychology, 10(3), 293-330.

Keller, H., \& Lamm, B. (2005). Parenting as expression of sociohistorical time: The case of German individualization. International Journal of Behaviour Development, 29(3), 238246.

Keller, H., Lamm, B., Abels, M., Yovsi, R., Borke, J., Jensen, H., Papaligoura, Z., Holub, C., Lo, W., Tomiyama, A. J., Su, Y., Wang, Y., \& Chaudhary, N. (2006). Cultural models, socialization goals, and parenting ethnotheories: A multicultural analysis. Journal of Cross-Cultural Psychology, 37(2), $155-172$.
Keller, H., Lohaus, A., Voelker, S., Elben, C., \& Ball, J. (2003). Warmth and contingency and their relationship to maternal attitudes toward parenting. The Journal of Genetic Psychology, 164(3), 275-292.

Keller, H., Yovsi, R., Borke, J., Kärtner, J., Jensen, H., \& Papaligoura, Z. (2004). Developmental consequences of early parenting experiences: Self-regulation and self-recognition in three cultural communities. Child Development, 75(6), 17451760.

Kobarg, A. P. R. (2006). Crenças e práticas de mães sobre o desenvolvimento infantil nos contextos rural e urbano. Dissertação de mestrado não-publicada, Universidade Federal de Santa Catarina, Florianópolis.

Meléndez, L. (2005). Parental beliefs and practices around early self-regulation: The impact of culture and immigra-tion. Infant and Young Children, 18(2), 136-146.

Piovanotti, M. R. (2007). Metas de socialização infantil e crenças sobre práticas de cuidador parental de mães primíparas. Dissertação de mestrado não-publicada, Universidade Federal de Santa Catarina, Florianópolis.

Ruela, S. F. (2006). Um estudo intergeracional de crenças valorizadas por mães em uma comunidade rural do Rio de Jáneiro. Dissertação de mestrado não-publicada, Universidade do Estado do Rio de Janeiro, Rio de Janeiro.

Seidl-de-Moura, M. L., Ribas Jr., R. C., Piccinini, C. A., Bastos, A. C. de S., Magalhães, C. M. C., Vieira, M. L., Salomão, N. M. R., Silva, A. M. R. M., \& Silva, A. K. (2004). Conhecimento sobre desenvolvimento infantil em mães primíparas de diferentes centros urbanos do Brasil. Estudos de Psicologia (Natal), 9(3), 421-429.

Sherbourne, C. D., \& Stewart, A. L. (1991). The MOS social support survey. Social Science \& Medicine, 32(6), 705-714.

Suizzo, M. A. (2002). French parents' cultural models and childrearing beliefs. International Journal of Behavioral Development, 26, 297-307.

Recebido: 20/03/2008 Última revisão: $23 / 10 / 2008$ Aceite final: 10/12/2008

\section{Sobre os autores:}

Gabriela Dal Forno Martins: Mestre em Psicologia, Programa de Pós-graduação em Psicologia, Universidade Federal de Santa Catarina.

Samira Mafioletti Macarini: Mestranda em Psicologia, Programa de Pós-graduação em Psicologia, Universidade Federal de Santa Catarina.

Virginia Azevedo Reis Sachetti: Professora Doutora e Coordenadora do Curso de Psicologia da Faculdade Metropolitana de Guaramirim.

Mauro Luís Vieira: Professor Doutor do Departamento de Psicologia da Universidade Federal de Santa Catarina.

Maria Lúcia Seidl-de-Moura: Professora Doutora Titular da Universidade do Estado do Rio de Janeiro.

Vera Silvia Raad Bussab: Professora Doutora Titular do Instituto de Psicologia, Universidade de São Paulo.

Endereço para correspondência: Departamento de Psicologia, Universidade Federal de Santa Catarina - Campus Universitário, Trindade - 88040-900 Florianópolis, SC. Endereço eletrônico: maurolvieira@gmail.com. 